\title{
COMAXIMIZABLE PRIMES
}

\author{
RAYMOND C. HEITMANN AND STEPHEN MCADAM
}

(Communicated by Louis J. Ratliff, Jr.)

\begin{abstract}
Let $P_{1}, \ldots, P_{n} \quad(n \geq 2)$ be not necessarily distinct nonzero prime ideals in the Noetherian, but not Henselian, domain $R$. We show that there is a finitely generated integral extension domain $T$ of $R$, containing distinct, pairwise comaximal prime ideals $Q_{1}, \ldots, Q_{n}$ lying over $P_{1}, \ldots, P_{n}$ respectively.
\end{abstract}

\section{INTRODUCTION}

In [HW, §1], W. Heinzer and S. Wiegand give a pleasant proof of the following interesting result.

(1.1) Theorem. Let $P$ be a nonzero prime ideal in a Noetherian domain $R$, and let $T$ be the integral closure of $R$ in the algebraic closure of the quotient field of $R$. Let $m$ be the number of primes in $T$ that lie over $P$. Then either $m=\infty$ or $m=1$. Furthermore, $m=1$ if and only if $R$ is a (local) Henselian domain, and $P$ is its unique maximal ideal.

The key step in their proof is the construction (in the case $m \neq 1$ ) of an integral extension domain of $R$ in which two distinct primes, $Q_{1}$ and $Q_{2}$, lie over $P$. We show that when $R$ is not Henselian, it is possible to force $Q_{1}$ and $Q_{2}$ to be comaximal. More generally, we consider the following idea.

Definition. Let $n \geq 2$, and let $P_{1}, \ldots, P_{n}$ be not necessarily distinct nonzero prime ideals in the domain $R$. We say that $P_{1}, \ldots, P_{n}$ are comaximizable if there is a finitely generated integral extension domain $T$ of $R$, containing distinct, pairwise comaximal primes $Q_{1}, \ldots, Q_{n}$ lying over $P_{1}, \ldots, P_{n}$ respectively.

We prove the following theorem.

(1.2) Theorem. For $n \geq 2$, let $P_{1}, \ldots, P_{n}$ be not necessarily distinct nonzero prime ideals in the Noetherian domain $R$. Then $P_{1}, \ldots, P_{n}$ are comaximizable if and only if $R$ is not a (local) Henselian domain.

Received by the editors April 9, 1990; presented at the 861st meeting of the AMS, Denton, Texas, November 2, 1990.

1980 Mathematics Subject Classification (1985 Revision). Primary 13B20, 13 A17. 
Throughout this paper, $R$ will be a domain, and $X$ will be an indeterminate over $R$. A domain with a unique maximal ideal will be called quasi-local. If it is also known to be Noetherian, we will call it local. We will freely use facts about integral extensions as in $[\mathrm{K}, \S 1-6]$. For background on Henselian domains, see [N, §43]. We specifically note the following terminology. When we say a domain is not Henselian, we will mean that it is not a quasi-local Henselian domain. In particular, a domain which is not quasi-local is automatically not Henselian.

\section{Preliminaries}

Definition. Let $P$ be a prime ideal in $R$, and let $Q$ be a prime ideal in $R[X]$ with $Q \cap R=P$, but with $Q \neq P R[X]$. Then we will call $Q$ an upper to $P$ in $R[X]$ (or more simply, an upper to $P$, or just an upper). (See [K, $\S 1-5]$.) If the upper $Q$ contains a monic polynomial, we will call $Q$ an integral upper.

Notice that if $K$ is an upper to zero in $R[X]$, then there is a natural embedding $R \subseteq R[X] / K$, making the domain $R[X] / K$ a simple extension of $R$. If $K$ is an integral upper, then $R[X] / K$ is a simple integral extension domain of $R$ since $K$ contains a monic polynomial.

(2.1) Remark. Let $R$ be an integrally closed domain. Then the integral uppers to zero in $R[X]$ are exactly the primes $K$ in $R[X]$ such that $K=f(X) R[X]$, with $f(X)$ a monic irreducible polynomial in $R[X]$. This fact is well known, and is easily proved starting with the (well-known) fact that if $\alpha$ is an element in some larger domain, and $\alpha$ is integral over $R$, then the minimal (monic irreducible) polynomial of $\alpha$ over the quotient field of $R$ is actually in $R[X]$.

(2.2) Lemma. Let $R \subseteq T$ be an integral extension of domains. If $L$ is an upper to zero in $T[X]$, then $K=L \cap R[X]$ is an upper to zero in $R[X]$. If $L$ is an integral upper, then $K$ is an integral upper.

Proof. As $R[X] \subseteq T[X]$ is an integral extension, and as $L \neq 0$, we have $K \neq 0$. Since $L \cap T=0$, we have $K \cap R=0$. Therefore, $K$ is an upper to zero in $R[X]$. Now suppose that $L$ is an integral upper. Then $T \subseteq T[X] / L$ is an integral extension. Thus $R \subseteq T[X] / L$ is an integral extension. Since $R \subseteq R[X] / K \subseteq T[X] / L$, we see that $R \subseteq R[X] / K$ is an integral extension, and thus $K$ must contain a monic polynomial and be an integral upper.

(2.3) Lemma. Let $g(X) \in B \in \operatorname{Spec} R[X]$, with $R$ a domain and with $g(X)$ a monic polynomial in $R[X]$. Then there is an upper to zero, $K$, in $R[X]$, with $g(x) \in K \subseteq B$.

Proof. Let $\bar{R}$ be the integral closure of $R$, and let $\bar{B}$ be a prime of $\bar{R}[X]$ lying over $B$. Let $F$ be the quotient field of $R$, and let $g(X)=g_{1}(X) \cdots g_{n}(X)$ be the prime factorization of $g(X)$ in $F[X]$. If $u_{i}$ is a root of $g_{i}(X) \quad(i=$ $1, \ldots, n)$, then $g\left(u_{i}\right)=0$, so that $u_{i}$ is integral over $\bar{R}$. As $g_{i}(X)$ is the minimal polynomial of $u_{i}$ over $F$, and as $\bar{R}$ is integrally closed, we know that $g_{i}(X) \in \bar{R}[X]$. Since $g(X) \in \bar{B}$, for some $i$ we must have $g_{i}(X) \in \bar{B}$. By 
(2.1), $g_{i}(X) \bar{R}[X]$ is an upper to zero in $\bar{R}[X]$ and is contained in $\bar{B}$. By (2.2), $g_{i}(X) \bar{R}[X] \cap R[X]$ is an upper to zero in $R[X]$, which clearly contains $g(X)$ and is contained in $B$. Let $K=g_{i}(X) \bar{R}[X] \cap R[X]$.

For the sake of reference, we state the next well-known lemma.

(2.4) Lemma. Let $S$ be a multiplicatively closed subset of the domain $R$, and let the domain $D$ be an integral extension of $R_{S}$. Then there is an integral extension domain $T$ of $R$, with $T_{S}=D$.

(2.5) Lemma. Let $R$ be the integral closure of a Noetherian domain, and let $L$ be a prime ideal of $R$. Then the integral closure of $R / L$ is the integral closure of a Noetherian domain (and so is a Krull domain).

Proof. Suppose that $R$ is the integral closure of the Noetherian domain $S$. Let $R^{\#}=R / L$, and let $S^{\#}=S /(L \cap S)$. By [N, (33.10)], $R^{\#}$ is an almost finite integral extension of $S^{\#}$. (That is, the quotient field of $R^{\#}$ is finitedimensional over the quotient field of $S^{\#}$.) Clearly we can find a domain $T^{\#}$ with $S^{\#} \subseteq T^{\#} \subseteq R^{\#}$, with $T^{\#}$ finitely generated over $S^{\#}$, and with the quotient field of $T^{\#}$ equal to the quotient field of $R^{\#}$. Thus $R^{\#}$ and $T^{\#}$ have the same integral closure. As $T^{\#}$ is a Noetherian domain, we are done.

(2.6) Lemma. Let $R$ be a domain that is not a field, and suppose that $\bar{R}$, the integral closure of $R$, is a Krull domain. Then in $R[X]$, there is a monic irreducible polynomial $\alpha(X)$, with degree $\alpha(X) \geq 2$.

Proof. Since $\bar{R}$ is not a field, it contains some $b \neq 0$ with $b$ a nonunit. Since $\bar{R}$ is a Krull domain, it enjoys the ascending chain condition on principal ideals. Therefore, we may choose our $b$ such that $b \bar{R}$ is as large as possible. Clearly $b$ is irreducible in $\bar{R}$, and hence is not the square of any element in $\bar{R}$. Thus $X^{2}-b$ is irreducible in $\bar{R}[X]$. Therefore, by $(2.1), L=\left(X^{2}-b\right) \bar{R}[X]$ is an integral upper to zero in $\bar{R}[X]$. By (2.2), LกR[X] is an integral upper to zero in $R[X]$. Let $\alpha(X)$ be a monic polynomial in $L \cap R[X]$, with degree $\alpha(X)$ as small as possible. Then clearly $\alpha(X)$ is irreducible in $R[X]$. Also, we must have degree $\alpha(X) \geq 2$, since $X^{2}-b$ divides $\alpha(X)$ in $\bar{R}[X]$. Thus $\alpha(X)$ satisfies the lemma.

(2.7) Lemma. Let $R$ be the integral closure of a Noetherian domain, and let $P$ be a nonzero prime ideal of $R$. Then the intersection of all powers of $P$ is zero.

Proof. Let $R$ be the integral closure of the Noetherian domain $S$, and let $u$ be an element in $P$ but in none of the other (finitely many) primes of $R$ that lie over $P \cap S[\mathrm{~N},(33.10)]$. Then we easily see that $P$ is the only prime of $R$ lying over $P \cap S[u]$. A well-known theorem of Chevalley [C] says that there is a discrete valuation ring $(V, N)$ between $S[u]$ and its quotient field, with $N \cap S[u]=P \cap S[u]$. Clearly $R \subseteq V$, and $N \cap R$ must equal $P$. As the intersection of all powers of $N$ is zero, the same must be true of the intersection of all powers of $P$. 
The next lemma is a key result of [HW, $§ 1]$. We offer a variation on the proof.

(2.8) Lemma [HW, (1.4)]. Let $R$ be an integrally closed domain, and suppose that $N$ and $H$ are distinct maximal ideals of $R$, with $H \neq H^{2}$. Then there is a simple integral extension domain of $R$ in which two distinct maximal ideals lie over $N$.

Proof. As $N$ and $H$ are comaximal, pick $a \in R$ with $a-1 \in N$ and $a \in H$. Let $y \in H-H^{2}$. Since $N$ and $H^{2}$ are comaximal, pick $b \in R$ with $b \in N$ and $b-y \in H^{2}$. Note that $b \in H-H^{2}$. Let $f(X)=X^{2}-a X+b$. We claim that $f(X)$ is irreducible in $R[X]$. If not, it would have a root $\alpha \in R$. Since $\alpha^{2}=a \alpha-b$ and $a, b \in H$, we have $\alpha \in H$. Since $b=a \alpha-\alpha^{2}$, and $a, \alpha \in H$, we have $b \in H^{2}$. This is a contradiction, which proves that $f(X)$ is irreducible. Let $K=f(X) R[X]$, so that $K$ is an integral upper to zero, by (2.1). Thus $R[X] / K$ is a simple integral extension domain of $R$. We claim that it contains two maximal ideals lying over $N$. Since $a \equiv 1$ $\bmod N$, and $b \in N, f(X) \equiv X^{2}-X \bmod N$. Thus $K=f(X) R[X]$ is contained in $(N, X) R[X]$ and in $(N, X-1) R[X]$. One can easily verify that $(N, X) R[X] / K$, and $(N, X-1) R[X] / K$ are distinct maximal ideals in $R[X] / K$, both of which lie over $N$.

(2.9) Corollary. Let $R$ be Noetherian domain that is not Henselian. Let $N$ be a maximal ideal of $\bar{R}$, the integral closure of $R$. Then $\bar{R}_{N}$ is not Henselian.

Proof. First, suppose that $N$ is the unique maximal ideal of $\bar{R}$, so that $\bar{R}=$ $\bar{R}_{N}$. Since the local domain $R$ is not Henselian, there is an integral extension domain $T$ of $R$ that is not quasi-local. Consider the composite $T \bar{R}$. Being an integral extension of $T$, it cannot be quasi-local. As it is also an integral extension of $\bar{R}$, we have that $\bar{R}=\bar{R}_{N}$ is not Henselian in this case. Next, suppose that $H$ is a maximal ideal of $\bar{R}$ distinct from $N$. By (2.7), $H^{2} \neq H$. By (2.8), there is an integral extension domain of $\bar{R}$ in which two distinct maximal ideals lie over $N$. Localizing, we see that there is an integral extension domain of $\bar{R}_{N}$ in which two distinct maximal ideals lie over $N_{N}$. Again, $\bar{R}_{N}$ is not Henselian.

\section{CoMAXIMIZABLE PRIMES}

(3.1) Proposition. Let $R$ be a Noetherian domain. Let $P$ and $Q$ be nonzero primes of $R$, and suppose there is a maximal ideal $M$ of $R$ with $P \subseteq M$ but $Q \nsubseteq M$. Then there is a simple integral extension domain $R^{\prime}$ of $R$, and primes $P^{\prime}$ and $Q^{\prime}$ of $R^{\prime}$ lying over $P$ and $Q$ respectively, such that $P^{\prime}$ and $Q^{\prime}$ are comaximal in $R^{\prime}$.

Proof. Suppose that in $R[X]$, we can find an integral upper $K$ to zero, with $K \subseteq(P, X) \cap(Q, X-1)$. Then $R^{\prime}=R[X] / K$ will be a simple integral extension of $R$, and $P^{\prime}=(P, X) / K$ and $Q^{\prime}=(Q, X-1) / K$ will be primes 
of $R^{\prime}$ lying over $P$ and $Q$. Since $P^{\prime}$ will contain the coset $X \bmod K$, and $Q^{\prime}$ will contain the coset $X-1 \bmod K$, no ideal of $R^{\prime}$ can contain both $P^{\prime}$ and $Q^{\prime}$, since such an ideal would then contain the coset $1 \bmod K$, which is the identity of $R^{\prime}$. Therefore, we need only find such a $K$.

We now lift our problem to the integral closure $\bar{R}$ of $R$. Let $\bar{P}$ and $\bar{Q}$ be primes of $\bar{R}$ lying over $P$ and $Q$ respectively. By the going up theorem, there is a maximal ideal $\bar{M}$ of $\bar{R}$ lying over $M$ with $\bar{P} \subseteq \bar{M}$. Clearly $\bar{Q} \nsubseteq \bar{M}$. Thus the hypotheses on $R, P, Q$, and $M$ lift to $\bar{R}, \bar{P}, \bar{Q}$, and $\bar{M}$, except that the hypothesis that $R$ is Noetherian is replaced with the fact that $\bar{R}$ is the integral closure of a Noetherian domain. Suppose now that we can find an integral upper $\bar{K}$ to zero in $\bar{R}[X]$, with $\bar{K} \subseteq(\bar{P}, X) \cap(\bar{Q}, X-1)$. Let $K=\bar{K} \cap R[X]$. By (2.2), $K$ will be an integral upper to zero in $R[X]$, and obviously $K$ will be contained in $(P, X) \cap(Q, X-1)$. Thus, we need only find $\bar{K}$.

We will return to our original unbarred notation, now assuming that $R$ is the integral closure of a Noetherian domain. By (2.1), we seek a monic polynomial $f(X)$ that is irreducible in $R[X]$, and satisfies $f(X) \in(P, X) \cap(Q, X-1)$. Since $Q$ and $M$ are comaximal, we can find an $a \in R$ with $a \equiv 0 \bmod M$ and $a \equiv 1 \bmod Q$. Thus $(Q, X-1)=(Q, X-a)$, and $a \in M$. Since (2.7) shows that the intersection of all powers of $M$ is zero, clearly $M P \neq P$; thus $P=M P+Q P$ implies $Q P \nsubseteq M P$. Choose $b \in Q P-M P$. Let $f(X)=$ $X^{2}-a X+b$. As $b \in P$, clearly $f(X) \in(P, X)$. As $b \in Q$, clearly $f(X) \in$ $(Q, X-a)=(Q, X-1)$. It only remains to show that $f(X)$ is irreducible in $R[X]$. If not, then write $f(X)=(X-c)(X-d)$. Since $c d=b \in P$, we may assume that $c \in P$. Now $a, c \in M$, and $a=c+d$ implies $d \in M$. Thus $b=c d \in M P$, which contradicts our choice of $b$. This shows that $f(X)$ is irreducible, and completes the proof.

(3.2) Remark. In (3.1), we can also arrange to have height $P^{\prime}=$ height $P$, and height $Q^{\prime}=$ height $Q$. We will show how to get the latter, the former working similarly. Since $Q^{\prime}=(Q, X-1) / K$, we want height $(Q, X-1) / K=$ height $Q$. Recall that via the second paragraph of the above proof, $K=$ $\bar{K} \cap R[X]$. Thus $R \subseteq R[X] / K \subseteq \bar{R}[X] / \bar{K}$ are integral extensions. Since $(\bar{Q}, X-1) / \bar{K}$ lies over $(Q, X-1) / K$, which in turn lies over $Q$, we always have height $Q \geq \operatorname{height}(Q, X-1) / K \geq$ height $(\bar{Q}, X-1) / \bar{K}$. If we can arrange to have height $(\bar{Q}, X-1) / \bar{K}=$ height $Q$, equality will hold throughout, and we will be done. However, since $\bar{R}$ is integrally closed, the integral extension $\bar{R} \subseteq \bar{R}[X] / \bar{K}$ satisfies the going down theorem, and so, since $(\bar{Q}, X-1) / \bar{K}$ lies over $\bar{Q}$, these two primes have the same height. Thus, we only need height $\bar{Q}=$ height $Q$. Of course we know this does hold for some $\bar{Q}$ lying over $Q$, completing the argument.

(3.3) Corollary. Let $R$ be a Noetherian domain that is not Henselian. Let $M$ be a maximal ideal of $R$ with height $M \geq 2$. Let $P_{1}, \ldots, P_{n}$ be nonzero prime ideals in $R$. Then there is a finitely generated integral extension domain $R^{\prime}$ 
of $R$, and primes $M^{\prime}$ and $P_{1}^{\prime}, \ldots, P_{n}^{\prime}$ in $R^{\prime}$ lying over $M$ and $P_{1}, \ldots, P_{n}$ respectively, such that height $M^{\prime} \geq 2$, and $P_{i}^{\prime} \nsubseteq M^{\prime}$, for $i=1, \ldots, n$.

Proof. We will induct on $n$. Suppose $n=1$, and let $P=P_{1}$. If $P \nsubseteq M$, we let $R^{\prime}=R$, and we are done. Suppose that $P \subseteq M$. Let $\bar{R}$ be the integral closure of $R$, and let $\bar{V}$ be a maximal ideal of $\bar{R}$ lying over $M$, with height $N=$ height $M \geq 2$. By (2.9), since $R$ is not Henselian, neither is $\bar{R}_{N}$. Thus there is an integral extension domain of $\bar{R}_{N}$ having at least two distinct maximal ideals lying over $N_{N}$, and so by (2.4) there is an integral extension domain $T^{\prime}$ of $\bar{R}$ having at least two distinct maximal ideals, $M_{1}^{\prime}$ and $M_{2}^{\prime}$, lying over $N$. Since $\bar{R}$ is integrally closed, the going down theorem shows that for $i=1,2$, height $M_{i}^{\prime}=$ height $N \geq 2$. Now pick $u \in M_{1}^{\prime}-M_{2}^{\prime}$. Let $T=R[u], M_{1}=M_{1}^{\prime} \cap T$, and $M_{2}=M_{2}^{\prime} \cap T$. Then $T$ is Noetherian, $M_{1}$ and $M_{2}$ lie over $M$ in $R, M_{1}$ and $M_{2}$ are distinct (since $u \in M_{1}-M_{2}$ ), and $M_{1}$ and $M_{2}$ both have height at least 2. Let $p$ be a prime of $T$ lying over $P$. If $p \nsubseteq M_{1}$, then we let $R^{\prime}=T, P^{\prime}=p$, and $M^{\prime}=M_{1}$, and we are done. Therefore, suppose that $p \subseteq M_{1}$. Then by (3.1) (applied in $T$, with $P=p$, $Q=M_{2}$, and $\left.M=M_{1}\right)$, there is a finitely generated integral extension domain $R^{\prime}$ of $T$, and comaximal primes $P^{\prime}$ and $M^{\prime}$ of $R^{\prime}$ lying over $p$ and $M_{2}$ respectively. By (3.2), we may assurne that height $M^{\prime}=$ height $M_{2} \geq 2$. Since $P^{\prime}$ and $M^{\prime}$ are comaximal, surely $P^{\prime} \nsubseteq M^{\prime}$. This $R^{\prime}, P^{\prime}$, and $M^{\prime}$ satisfy the corollary (when $n=1$ ).

Suppose now that for $n>1$, we know the corollary is true for $n-1$. Then applying the case $n-1$ to $M$ and $P_{1}, \ldots, P_{n-1}$, we can find a finitely generated integral extension domain $R^{\#}$ of $R$, and primes $M^{\#}, P_{1}^{\#}, \ldots, P_{n-1}^{\#}$ lying over $M, P_{1}, \ldots, P_{n-1}$ respectively, with height $M^{\#} \geq 2$ and $P_{i}^{\#} \nsubseteq M^{\#}$ for $i=1, \ldots, n-1$. Let $P_{n}^{\#}$ be any prime of $R^{\#}$ lying over $P_{n}$. Apply the case $n=1$ to $R^{\#}, M^{\#}$, and $P_{n}^{\#}$, and find a finitely generated integral extension domain $R^{\prime}$ of $R^{\#}$, with primes $M^{\prime}$ and $P_{n}^{\prime}$ lying over $M^{\#}$ and $P_{n}^{\#}$, and with height $M^{\prime} \geq 2$ and $P_{n}^{\prime} \nsubseteq M^{\prime}$. Now let $P_{1}^{\prime}, \ldots, P_{n-1}^{\prime}$ be any primes of $R^{\prime}$ lying over $P_{1}^{\#}, \ldots, P_{n-1}^{\#}$ respectively. Since for $i=1, \ldots, n-1$ we have $P_{i}^{\#} \nsubseteq M^{\#}$, surely we must have $P_{i}^{\prime} \nsubseteq M^{\prime}$. The corollary is clearly satisfied, and we are done.

(3.4) Proposition. Let $R$ be a Noetherian domain. Let $P$ and $Q$ be nonzero prime ideals of $R$. (Here, we allow the possibility that $P=Q$.) Suppose there is a maximal ideal $M$ of $R$ such that $P \nsubseteq M, Q \nsubseteq M$, and height $M \geq 2$. Then there is a simple integral extension domain $R^{\prime}$ of $R$, and distinct primes $P^{\prime}$ and $Q^{\prime}$ of $R^{\prime}$ lying over $P$ and $Q$ respectively, such that $P^{\prime}$ and $Q^{\prime}$ are comaximal in $R^{\prime}$.

Proof. As in the proof of (3.1), we need only find an integral upper $K$ to zero in $R[X]$ with $K \subseteq(P, X) \cap(Q, X-1)$. As in that proof, we may also lift to the integral closure of $R$ (here using the fact that the integral closure of $R$ 
contains a maximal ideal lying over $M$ and having the same height as $M$ ). Thus, we will assume that $R$ is the integral closure of a Noetherian domain. By (2.1), we seek a monic polynomial $f(X)$ that is irreducible in $R[X]$, and satisfies $f(X) \in(P, X) \cap(Q, X-1)$.

Since $P \nsubseteq M$ and $Q \nsubseteq M$, we have $M$ and $P \cap Q$ comaximal. Write $1=x+y$, with $x \in P \cap Q$ and $y \in M$. Let $L$ be a prime of $R$ minimal over $y$, with $L \subseteq M$. Since $1=x+y$, we see that $L$ and $P \cap Q$ are comaximal. Since $R$ is a Krull domain, height $L=1<$ height $M$, so that $L$ is not maximal. Thus $R / L$ is not a field, and by (2.5), its integral closure is a Krull domain. Using (2.6), let $\alpha(x)=X^{m}+\alpha_{m-1} X^{m-1}+\cdots+\alpha_{0}$ be a monic irreducible polynomial in $(R / L)[X]$ with $m \geq 2$. For $i=0,1, \ldots, m-1$, let $b_{i} \in R$ be a coset representative of $\alpha_{i}$. Since $L$ and $P \cap Q$ are comaximal, for $i=0,1, \ldots, m-1$, use the Chinese Remainder Theorem to find $c_{i} \in R$ with $c_{i} \equiv b_{i} \bmod L$, and $c_{i} \equiv 0 \bmod P \cap Q$. Thus $c_{i} \in P \cap Q$, and $c_{i}$ is a coset representative for $\alpha_{i}$. Since $L$ and $Q$ are clearly comaximal, pick $a \in R$, with $a \equiv 0 \bmod L$ and $a \equiv 1 \bmod Q$. Thus $a \in L$ and $(Q, X-1)=(Q, X-a)$. We now define $f(X)$ to be $X^{m-1}(X-a)+c_{m-1} X^{m-1}+\cdots+c_{0}$. Since each $c_{i} \in P$, and since $m-1 \geq 1$, we see immediately that $f(X) \in(P, X)$. Since each $c_{i} \in Q$, we also see that $f(X) \in(Q, X-a)=(Q, X-1)$. It only remains to show that $f(X)$ is irreducible in $R[X]$. Surely it will suffice to show that $f(X)$ taken modulo $L$ is irreducible in $(R / L)[X]$. However, we claim that $f(X) \bmod L$ is just the irreducible polynomial $\alpha(X)$. This is easily seen, since $a \in L$, and since for $i=0, \ldots, m-1, c_{i} \bmod L$ is $\alpha_{i}$. This completes the proof.

We now prove our main result, (1.2).

Proof of (1.2). Since any integral extension domain of a Henselian domain is quasi-local, one direction is obvious. For the other direction, assume that $R$ is not Henselian. We first treat the case in which $R$ contains a maximal ideal $M$, with height $M \geq 2$. By (3.3), it does no harm to assume that $M$ does not contain any of $P_{1}, \ldots, P_{n}$. Fix an algebraic closure $\Omega$ of the quotient field of $R$ and without loss, assume that all extensions of $R$ that we mention come from $\Omega$. Consider all possible ordered $n+1$-tuples $\left(T, Q_{1}, \ldots, Q_{n}\right)$ where $T$ is a finitely generated integral extension domain of $R$, and $Q_{1}, \ldots, Q_{n}$ are primes of $T$ lying over $P_{1}, \ldots, P_{n}$ respectively. Our task is to find such an $n+1$-tuple such that whenever $i \neq k$, we have $Q_{i}$ and $Q_{k}$ comaximal (and hence distinct $)$ in $T$. For each such $n+1$-tuple, consider the set $\left\{(i, k) \mid Q_{i}\right.$ and $Q_{k}$ are comaximal in $\left.T\right\}$. Let $\left(T, Q_{1}, \ldots, Q_{n}\right)$ be a fixed $n+1$-tuple chosen so as to make the size of the set just mentioned as large as possible. We claim that in fact $Q_{i}$ and $Q_{k}$ are comaximal whenever $i \neq k$, so that this $n+1$-tuple is the one we seek. Assume this is not so. We will derive a contradiction. For some $i \neq k, Q_{i}$ and $Q_{k}$ are assumed not comaximal. Reordering if necessary, we may say that $Q_{1}$ and $Q_{2}$ are not comaximal. Let $N$ be a maximal ideal of $T$ lying over $M$, with height $N=$ height $M \geq 2$. 
Since $M$ does not contain $P_{1}$ or $P_{2}$, clearly $N$ does not contain $Q_{1}$ or $Q_{2}$. By (3.4), there is a finitely generated integral extension domain $T^{\prime}$ of $T$, with primes $Q_{1}^{\prime}$ and $Q_{2}^{\prime}$ lying over $Q_{1}$ and $Q_{2}$ respectively, and with $Q_{1}^{\prime}$ and $Q_{2}^{\prime}$ comaximal. Let $Q_{3}^{\prime}, \ldots, Q_{n}^{\prime}$ be any primes of $T^{\prime}$ lying over $Q_{3}, \ldots, Q_{n}$ respectively. Note that if for some $i \neq k$ we have $Q_{i}$ and $Q_{k}$ comaximal in $T$, then automatically we have $Q_{i}^{\prime}$ and $Q_{k}^{\prime}$ comaximal in $T^{\prime}$. Thus, $\left\{(i, k) \mid Q_{i}\right.$ and $Q_{k}$ are comaximal in $\left.T\right\} \subseteq\left\{(i, k) \mid Q_{i}^{\prime}\right.$ and $Q_{k}^{\prime}$ are comaximal in $\left.T^{\prime}\right\}$. By the maximality of our choice $\left(T, Q_{1}, \ldots, Q_{n}\right)$, we must have that these two sets are equal. On the other hand, we have $(1,2)$ in the second of these sets, but not in the first. This contradiction completes the argument for this case.

The remaining case is when every maximal ideal of $R$ has height 1 . In particular, this means that each of the primes $P_{1}, \ldots, P_{n}$ is maximal. Let $T^{*}$ be the integral closure of $R$ in $\Omega$. By (1.1), we see that in $T^{*}$, there are distinct primes $Q_{1}^{*}, \ldots, Q_{n}^{*}$ lying over $P_{1}, \ldots, P_{n}$ respectively. For $1 \leq i \leq n$, pick $u_{i}$ in $Q_{i}^{*}$ but in none of the rest of $Q_{1}^{*}, \ldots, Q_{n}^{*}$. Let $T=R\left[u_{1}, \ldots, u_{n}\right]$, and let $Q_{i}=Q_{i}^{*} \cap T$. We see that $T$ is finitely generated over $R$, and that $Q_{1}, \ldots, Q_{n}$ are distinct primes of $T$ lying over $P_{1}, \ldots, P_{n}$ respectively. Being distinct and maximal assures that $Q_{1}, \ldots, Q_{n}$ will be pairwise comaximal. Thus $P_{1}, \ldots, P_{n}$ are comaximizable, and we are done.

As a corollary, we strengthen (1.1) in the case that $R$ is not Henselian.

(3.5) Corollary. Let $P$ be a nonzero prime ideal in the Noetherian, but not Henselian, domain $R$. Let $T$ be the integral closure of $R$ in the algebraic closure of the quotient field of $R$. Then in $T$, there is an infinite set of distinct, pairwise comaximal primes $P_{1}, P_{2}, P_{3}, \ldots$, all lying over $P$.

Proof. We will inductively construct a chain of rings, $R \subseteq R_{1} \subseteq R_{2} \subseteq R_{3} \subseteq$ $\cdots \subseteq T$, such that for $m \geq 1, R_{m}$ will be a finitely generated extension of $R$, and will contain distinct primes $Q_{m 1}, \ldots, Q_{m m}$ all lying over $P$. Furthermore, if $m \geq 2$, then $Q_{m 1}, \ldots, Q_{m m}$ will be pairwise comaximal, and we will have $Q_{m k} \cap R_{m-1}=Q_{(m-1) k}$ for $1 \leq k \leq m-1$, while $Q_{m m} \cap R_{m-1}=Q_{(m-1)(m-1)}$. Suppose that this chain has been constructed. Let $D=\bigcup R_{m}, 1 \leq m \leq \infty$. Also, for any $m \geq 1$, let $Q_{m}=\bigcup Q_{i m}, m \leq i \leq \infty$. The reader can easily verify that $D$ is a domain between $R$ and $T$, and that $Q_{1}, Q_{2}, Q_{3}, \ldots$ are distinct, pairwise comaximal, and lie over $P$. Thus, if $P_{1}, P_{2}, P_{3}, \ldots$ are any primes of $T$ lying over $Q_{1}, Q_{2}, Q_{3}, \ldots$ respectively, then $P_{1}, P_{2}, P_{3}, \ldots$ are seen to satisfy the conclusion of the corollary.

It only remains to construct the $R_{m}$ and $Q_{m 1}, \ldots, Q_{m m}$. Let $R_{1}=R$ and $Q_{11}=P$, and inductively suppose that for $m-1 \geq 1$, we already have $R_{m-1}$, and $Q_{(m-1) 1}, \ldots, Q_{(m-1)(m-1)}$. Since $R_{m-1}$ is finitely generated over $R$, it is Noetherian. We claim that $R_{m-1}$ is not Henselian. If $m-1=1$, it is given that $R_{1}=R$ is not Henselian. If $m-1 \geq 2$, then since $Q_{(m-1) 1}, \ldots, Q_{(m-1)(m-1)}$ are distinct and pairwise comaximal, clearly $R_{m-1}$ 
is not local, and so is not Henselian. We apply (1.2) to $R_{m-1}$ and the list of primes $Q_{(m-1) 1}, Q_{(m-1) 2}, \ldots, Q_{(m-1)(m-2)}, Q_{(m-1)(m-1)}, Q_{(m-1)(m-1)}$. (Here, note that the last two primes in our list are identical.) By (1.2), there is a finitely generated integral extension domain $R_{m}$ of $R_{m-1}$ and a list of distinct, pairwise comaximal primes $Q_{m 1}, \ldots, Q_{m m}$ of $R_{m}$ lying respectively over the corresponding primes in our first list. This completes the proof.

We leave to the reader the proof of the next corollary, a variation on the Chinese Remainder Theorem.

(3.6) Corollary. Let $R$ be a Noetherian, but not Henselian, domain. Let $P_{1}, \ldots, P_{n}$ be not necessarily distinct nonzero prime ideals in $R$, and let $a_{1}, \ldots$, $a_{n}$ be elements of $R$. Then there is an irreducible monic polynomial $f(X)$ in $R[X]$, such that $f\left(a_{i}\right) \in P_{i}$ for $1 \leq i \leq n$.

\section{REFERENCES}

[C] C. Chevalley, La notion d'anneau de decomposition, Nagoya J. Math. 7 (1954), 21-33.

[HW] W. Heinzer and S. Wiegand, Prime ideals in two-dimensional polynomial rings, Proc. Amer. Math. Soc. 107 (1989), 577-586.

[K] I. Kaplansky, Commutative rings, Univ. of Chicago Press, Chicago, 1974.

[N] M. Nagata, Local rings, Wiley Interscience, New York, 1962.

Department of Mathematics, University of Texas, Austin, TeXas 78712 